\title{
Glutamine Addiction: An Achilles Heel for MYC-Overexpressing Breast Cancer
}

\author{
E. Jiang ${ }^{1}$ and A. Waldron ${ }^{1}$ \\ 1. Bergen County Academies, Nano-Structural Imaging Lab, 200 Hackensack Avenue, Hackensack, NJ \\ 07601
}

MYC and PI3K are oncogenes that, once deregulated, can alter cell metabolism to support growth and survival [1]. In particular, MYC is frequently overexpressed or mutated in breast cancer [2]. MYC overexpression is known to cause glutamine addiction by reprogramming cellular metabolism [1]. Importantly, glutamine deprivation can induce apoptotic cell death in glutamine-addicted cancer cells [3]. In this study, it was hypothesized that glutamine deprivation would induce apoptosis in MCF10A mammary epithelial cells overexpressing MYC. Wild type, PI3K mutant, and MYC-overexpressing MCF10A human mammary epithelial cells were subjected to glutamine deprivation for 48 hours, stained with the nucleic acid stain DAPI, and imaged using fluorescence microscopy. A western blot protein immunoblot assay was also applied to the cell lines to confirm MYC overexpression. An MTS cell viability colorimetric assay was performed for quantitative analysis of cell viability under glutamine deprivation, and an apoptotic cell death luminescence assay was performed for quantitative analysis of cell death under glutamine deprivation. Flow cytometry was used to measure cell death relative to total cell population.

Results via microscopy qualitatively demonstrated that glutamine deprivation induced significant cell death in MYC-overexpressing, but not in wild-type or PI3K mutant MCF10A cells. Results from the MTS cell viability assay demonstrated that glutamine deprivation significantly reduced the viability of a MYC-overexpressing MCF10A cell population $(\mathrm{p}<0.003)$. This result was confirmed by a luminescence-based caspase assay, which showed a significant increase in apoptosis in MYCoverexpressing MCF10A cells from full to glutamine- deprived medium conditions $(p<0.05)$. Additionally, results from flow cytometry indicated that pharmacological inhibitors of glutamine use induced potent cell death for MYC-overexpressing cells. Surprisingly, total amino acid deprivation did not induce significant cell death, warranting future mechanistic study.

The results of this study showed that glutamine deprivation or pharmacological inhibition of glutaminolysis induces apoptosis in MYC-overexpressing MCF10A cells, but not in the other two cell types. These results are consistent with previous publications showing that $\mathrm{MYC}$ is the master regulator of glutaminolysis, and that MYC overexpression may render cancer cells addicted to glutaminolysis [1, 3]. Taken together, this investigation demonstrates the potential efficacy of glutaminolysis-targeting in treating cancers with MYC overexpression, and provides a basis for further inquiry.

\section{References:}

[1] Wise D, et al. Myc regulates a transcriptional program that stimulates mitochondrial glutaminolysis and leads to glutamine addiction. PNAS. 2008 Dec 2; 105(48), 182-7.

[2] Gao, J, et al. (2013). Integrative Analysis of Complex Cancer Genomics and Clinical Profiles Using the cBioPortal. Science Signaling, 6(269).

[3] Wise D and Thompson C. Glutamine addiction: a new therapeutic target in cancer. Trends Biochem Sci. 2010 Aug; 35(8), 427-33. 


\section{MCF10A Wild-type}
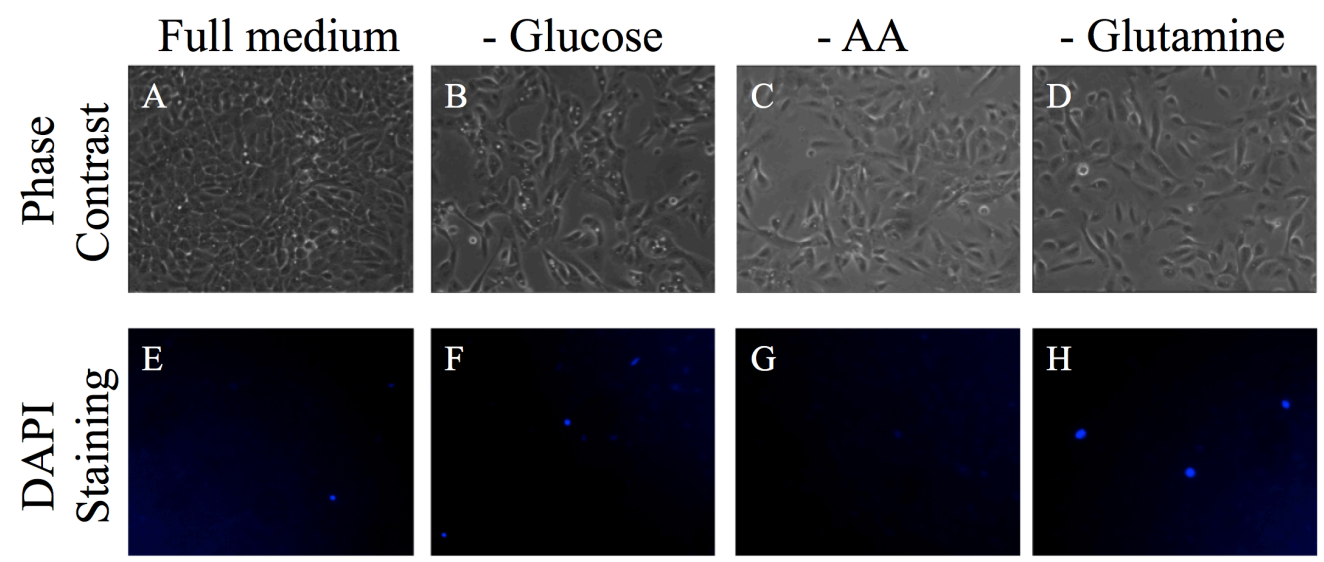

Figure 1. Imaging of wild-type MCF10A cells. (A-D) Fully confluent in full medium, and slightly less so in other media conditions (E-H) DAPI staining shows insignificant cell death in all media conditions.

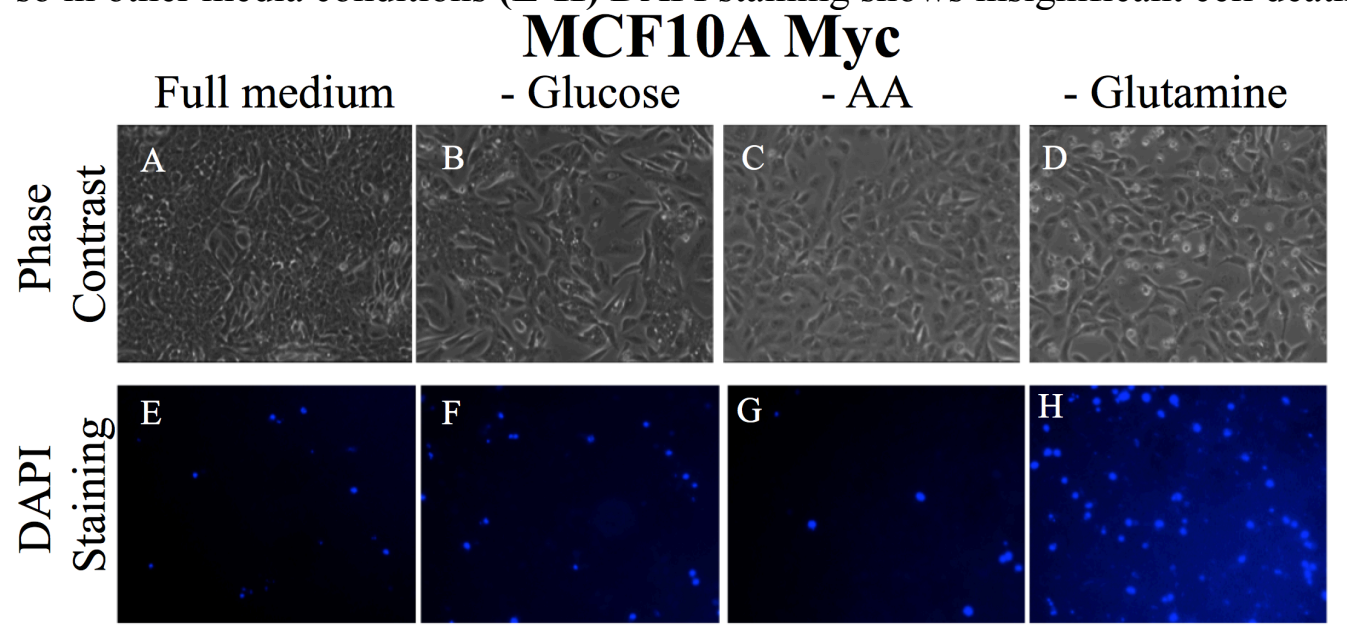

Figure 2. Imaging of Myc-overexpressing MCF10A cells. (A) Cells are fully confluent in full medium. (B-D) Cells are somewhat less confluent in other media. (E-F) Insignificant cell death is observed. (G) Significant cell death is observed in medium lacking glutamine.

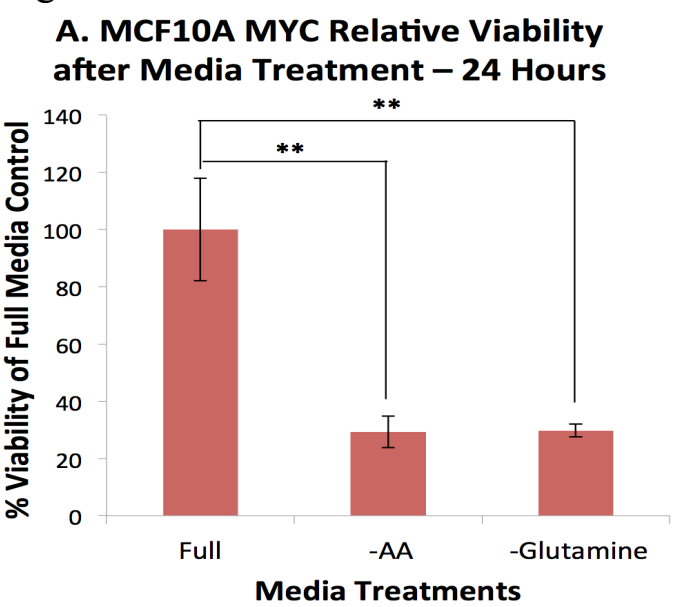

\section{B. MCF10A MYC Relative Cell Death after Media Treatment - 24hr}

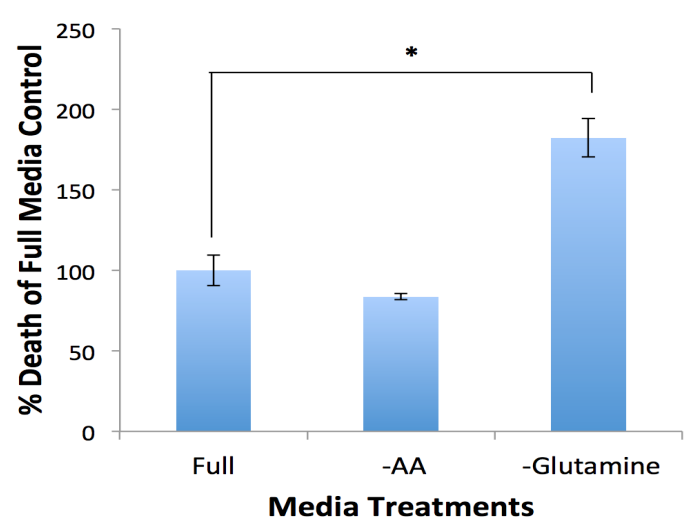

Figure 3. Quantitative assays performed on Myc-overexpressing MCF10A cells. (A) MTS cell viability assay shows that both media conditions reduce viability $(\mathrm{p}<0.01)$. (B) Luminescence-based assay shows that glutamine deprivation induces potent apoptosis, but total amino acid deprivation does not $(\mathrm{p}<0.05)$. 